\title{
Launch Velocities in Successful Golf Putting: An Analytical Analysis
}

\author{
John F. Mahoney \\ Department of Industrial and Systems Engineering, University of Florida, Gainesville, FL, 32611, USA \\ E-mail: mahoney@ise.ufl.edu \\ Daniel P. Connaughton (Corresponding author) \\ College of Health and Human Performance, University of Florida, P.O. Box 118200, Gainesville, FL, 32611, USA \\ E-mail: danc@hhp.ufl.edu
}

Received: 11-02- 2017

doi:10.7575/aiac.ijkss.v.5n.2p.24
Accepted: 22-04- 2017

Published: 30-04- 2017

URL: http://dx.doi.org/10.7575/aiac.ijkss.v.5n.2p.24

\begin{abstract}
Background: This study is concerned with the special case of a putted ball intersecting a standard golf hole at its diameter. The velocity of the ball at the initial rim of the hole is termed the launch velocity and depending upon its value the ball may either be captured or it may escape capture by jumping over the hole. The critical value of the launch velocity $(V)$ is such that lesser values result in capture while greater values produce escape. Purpose: Since the value of the $V$ entered prominently in some theoretical studies of putting, the aim of the current study is to provide an original re-evaluation of $V$ and to contrast our results with existing results. Method: This analytical analysis relies on trigonometry in conjunction with Newtonian mechanics and the mathematics of projectiles. The results of a recent study into the mathematics of a bouncing ball which included the notions of restitution and friction were also employed in the analysis. Results: If bouncing and slipping do not occur when the ball hits the far rim of the hole our analysis produces a value of $V$ of $1.356 \mathrm{~m} / \mathrm{s}$. When bouncing and slipping are present we find that $V$ is at least $1.609 \mathrm{~m} / \mathrm{s}$ but increases beyond this value as slipping and friction become greater. Useful relations which relate the dynamics and geometry of the ball to $V$ are provided. Conclusion: Since ambient conditions may influence the extent of bounce and slippage we conjecture that the value of $V$ is not unique.
\end{abstract}

Keywords: golf, putting, ball velocities.

\section{Introduction}

Putting is acknowledged to be the most prevalent shot in golf with average and professional golfers taking approximately $40 \%$ of their strokes on the putting green (Alexander \& Kern, 2005; Broadie, 2012; Pelz, 2000; Wiren, 1992). This provides impetus for the study of putting from various points of view. The details of how a putted golf ball interacts with a regulation golf hole is of theoretical interest. The current investigation deals exclusively with the special case that the putted ball intersects the hole at its widest point - that is, the ball proceeds into the gap along the hole's diameter and not along any shorter chord. Of particular significance is the quantity $V$, termed the critical launch velocity. This is the velocity of the ball, as measured at the initial rim of the hole, so that balls with greater velocity jump over the hole, while balls of lesser velocity fall into the hole. Previous work concerning the value of $V$ is scant. Mahoney (1982, Eq. 3) proposed (after slight rearrangement) the relation

$$
V=(D / d) \sqrt{d g}
$$

where $d$ is the diameter of the ball (1.68 in.), $D$ is the diameter of the hole (4.25 in.), and $\mathrm{g}$ is the gravitational acceleration $\left(9.8 \mathrm{~m} / \mathrm{s}^{2}\right)$. The resulting value of $V$ is $1.636 \mathrm{~m} / \mathrm{s}$. This value is subject to suspicion owing to the manner in which (1) was derived. In the derivation it was assumed that during its flight above the hole that the ball was replaced by its point-mass. This has the effect of avoiding any controversy concerning the manner in which the ball and the far rim interact. By having the ball shrink to a point-mass thorny questions concerning the influence of the ball's rotation and whether the collision with the far rim is elastic or inelastic become moot. Details of the work by Holmes (1991) on the subject are not transparent. Holmes (1991) is an investigator whose research concerns the interaction of a putted ball with the hole. In that work he put forth the value of $V$ as $1.626 \mathrm{~m} / \mathrm{s}$, although the details of how that number was obtained are not clear. It seems evident that it should be possible to derive an equation (or system of equations) whose solution yields $V$. In contrast, Holmes (1991) presents a few equations, none of which contain $V$, along with a flow chart of alternative outcomes, and then in conjunction with a hidden computer program comes up with the stated value of $V$. The related assumptions were that the ball does not bounce nor slip upon contact with the far rim of the hole. Note that the $1.626 \mathrm{~m} / \mathrm{s}$ value advocated by Holmes (1991) is quite close to the 
value $1.636 \mathrm{~m} / \mathrm{s}$ given by (1). Furthermore, experimental data displayed by Holmes (1991, Fig. 14) seem to support the preceding numerical results, although the data represented in the figure exhibit considerable scatter.

To many the matter seems to be closed, but the prior assumptions that the ball does not bounce nor slip when it hits the far rim of the hole are open to question. On that basis we continue to explore the subject using less restrictive assumptions. Therefore, the aim of the current study is to provide an original re-evaluation of $V$ and to contrast our results with existing (Holmes, 1991) results. This study employs an analytical analysis that relies on trigonometry in conjunction with Newtonian mechanics and the mathematics of projectiles. The following section presents kinematic results, which are relevant, and have not appeared in the literature.

\section{Kinematic Considerations}

Consider a putted ball that is directed across the diameter of a regulation hole. As the ball leaves the initial rim of the hole its horizontal velocity is given as $v$. If $v$ is less than $v_{m}$ the ball will not reach the far rim, but will merely fall into the hole. Such putts are of no further concern. Putts for which $v$ exceeds $v_{m}$ will hit the far rim. It is this class of putts which is of interest. The situation is illustrated in Figure 1.

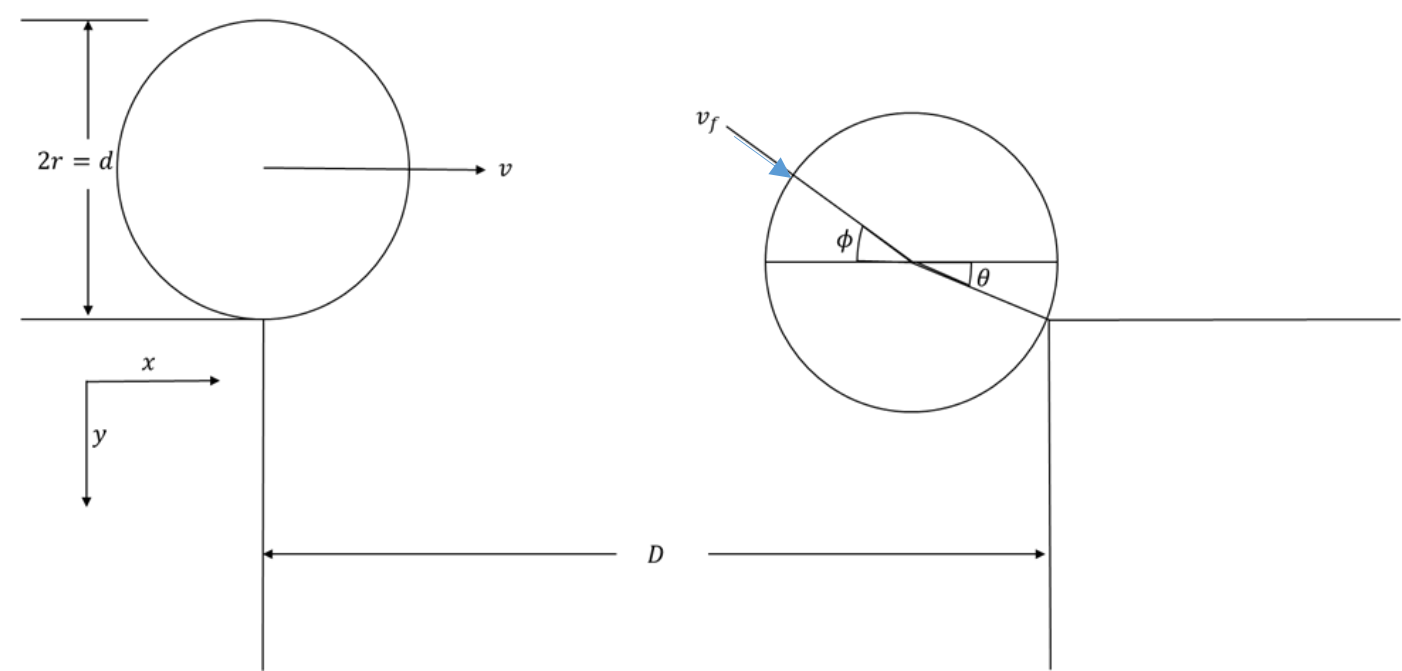

Figure 1. Depiction of a golf ball that leaves the initial rim (left) with a horizontal velocity $v$ and after an interval $t$ strikes the far rim (right) with a descending velocity $v_{f}$. The angle $\theta$ describes the orientation of the ball to the rim at impact while $\phi$ gives the downward motion of the ball at impact

Figure 1 is useful in explaining the two important angles $\theta$ and $\phi$. The first of these angles $\theta$ is the degree of declination (as measured from the horizontal) of the ball's radius which makes instantaneous contact with the far rim. This angle could vary from zero to 90 degrees and describes the orientation of the ball at the moment of contact.

The angle $\phi$ (again measured from the horizontal) describes the descending trajectory of the ball as it intersects with the far rim. It is also a measure of the slope of the terminal velocity vector. The symbolic notation used here and subsequently is summarized in Table 1.

Table 1. Nomenclature

\begin{tabular}{cl}
\hline$a$ & Acceleration experienced by ball upon impact \\
$d$ & Diameter of ball \\
$D$ & Diameter of hole \\
$e$ & Coefficient of restitution \\
$F$ & Force of impact \\
$g$ & Acceleration of gravity \\
$I$ & Moment of inertia of ball \\
$m$ & Mass of ball \\
$r$ & Radius of ball \\
$t$ & Time of passage from rim to rim \\
$v$ & Launch velocity of ball from initial rim \\
$v_{f}$ & Ball velocity upon reaching far rim \\
$v_{m}$ & Minimum launch velocity to reach far rim
\end{tabular}




$\begin{array}{cl}v_{x} & \text { Horizontal component of velocity } \\ v_{y} & \text { Downward component of velocity } \\ v_{\xi 1} & \text { Component of } v_{f} \text { in the } \xi \text { direction } \\ v_{\eta 1} & \text { Component of } v_{f} \text { in the } \eta \text { direction } \\ v_{\xi 2} & \text { Rebound velocity in the } \xi \text { direction } \\ v_{\eta 2} & \text { Rebound velocity in the } \eta \text { direction } \\ V & \text { Critical value of } v \\ x & \text { Horizontal dimension of length } \\ y & \text { Downward dimension of length } \\ \alpha & \text { Angular acceleration experienced by ball upon impact } \\ \epsilon & \text { Effective time of impact } \\ \eta & \text { Radial dimension of length } \\ \theta & \text { Angle giving orientation of ball at impact } \\ \theta_{1} & \text { Angle of approach to } \xi-\text { axis } \\ \theta_{2} & \text { Angle of rebound to } \xi-\text { axis } \\ \mu & \text { Coefficient of sliding function } \\ \xi & \text { Tangential dimension of length } \\ \tau_{1} & \text { Moment produced by } F_{\xi} \\ \tau_{2} & \text { Moment associate with } \alpha \\ \phi & \text { Angle giving descending trajectory of ball at impact } \\ \omega & \text { Angular velocity of ball }\end{array}$

The coordinate system adopted has $\mathrm{x}$ increasing horizontally (in Figure 1) from left to right, and $\mathrm{y}$ increasing downward. Let $t$ be the interval of time required for the ball to pass from rim to rim. The ball is assumed to behave ballistically and so from the physics of projectiles it follows that the components of the terminal velocity vector are

$$
v_{x}=v
$$

and

$$
v_{y}=g t
$$

The distances traveled in the coordinate directions are

$$
x=v t
$$

and

$$
y=g t^{2} / 2
$$

From the geometry displayed in Figure 1 it is clear that at the point of contact with the far rim that the center of the ball is short of the far rim by a horizontal distance $r \cos \theta$, and is above that rim by $r \sin \theta$. Hence, in its passage from rim to rim the ball moves horizontally by an amount

$$
x=D-r \cos \theta
$$

and drops by

$$
y=r-r \sin \theta
$$

Equating (4) to (6) followed by division by $r$ yields

$$
v t / r=D / r-\cos \theta
$$

Equating (5) and (7) followed by division by $r / 2$ gives

$$
g t^{2} / r=2(1-\sin \theta)
$$

Division of (9) by (8) gives

$$
g t / v=2(1-\sin \theta) /(D / r-\cos \theta)
$$

The slope of the terminal velocity vector is $\tan \phi$, and from (2) and (3)

$$
\tan \phi=g t / v
$$

From (10) to (11)

$$
\tan \phi=2(1-\sin \theta) /(D / r-\cos \theta)
$$

This important equation provides the relationship between $\theta$ and $\phi$.

Next, take the square root of both sides of (9) and then divide the result into (8) to get

$$
v / \sqrt{g r}=(D / r-\cos \theta) /(\sqrt{2} \sqrt{1-\sin \theta})
$$

This relates $\theta$ and $v$. 
Recall from the derivation of (1) that once the ball was in flight over the hole it was allowed to shrink to its center point and then continue its journey until it hit the rim on the far side of the hole. All of the equations (2) through (13) may be recast for this situation. The result of doing this is obtained by merely replacing the trigonometry functions (both $\cos \theta$ and $\sin \theta$ ) by zero as they appear in (2) through (13). In particular, the new version of (13) becomes

$$
V / \sqrt{g r}=D / \sqrt{2} r
$$

Upon noting that $d=2 r$ and doing simple rearrangement this becomes the same as (1).

\section{The Numerical Value of $v_{m}$}

The smallest value of $v$ which results in a ball making contact with the far rim is called $v_{m}$. The condition occurs when $\theta=0$. As previously mentioned $g=9.8 \mathrm{~m} / \mathrm{s}^{2}, D=4.25 \mathrm{in}$., and $d=1.68 \mathrm{in}$. It follows that $r=0.84 \mathrm{in}$., $D / r=$ 5.0595 , and in SI units $\sqrt{g r}=0.4573 \mathrm{~m} / \mathrm{s}$. Substitution into (13) produces $v_{m}=1.313 \mathrm{~m} / \mathrm{s}$, which agrees exactly with the value given by Holmes (1991). Although not called for, substitution into (12) reveals that $\tan \phi=0.4927$, and so $\phi=26.23$ degrees.

\section{Ball Velocities}

As the ball approaches the far rim it possesses two types of motion - it is translating and it is rotating. The center of the ball has a velocity $v_{f}$ (see Figure 1 ) which is given by

$$
v_{f}=v / \cos \phi
$$

Also, if the ball had been rolling as it left the initial rim, it would retain the angular velocity which is

$$
\omega=v / r
$$

Although this is likely to be the case it should be stated that putts which originate close to the initial rim might not have had the opportunity to develop a full measure of rotation.

Table 2 presents some numerical values of $\theta, \phi, v$, and $\omega$ that are generated by (12), (13), (14), and (15).

Table 2. A short table of values generated by (12), (13), (14), and (15). $\theta$ and $\phi$ are measured in degrees, $v$ and $\mathrm{v}_{\mathrm{f}}$ are expressed in meters per second, and $\omega$ is given in radians per second.

\begin{tabular}{ccccc}
\hline$\theta$ & $\phi$ & $v$ & $v_{f}$ & $\omega$ \\
\hline 0 & 26.23 & 1.313 & 1.463 & 61.539 \\
5 & 24.19 & 1.375 & 1.508 & 64.445 \\
10 & 22.08 & 1.449 & 1.564 & 67.913 \\
15 & 19.91 & 1.538 & 1.625 & 72.085 \\
20 & 17.71 & 1.642 & 1.724 & 76.959 \\
25 & 15.54 & 1.767 & 1.834 & 82.818 \\
30 & 13.41 & 1.918 & 1.971 & 89.895 \\
35 & 11.37 & 2.100 & 2.142 & 98.425 \\
40 & 9.45 & 2.323 & 2.355 & 108.877 \\
45 & 7.67 & 2.601 & 2.624 & 121.907 \\
50 & 6.05 & 2.953 & 2.969 & 138.405 \\
55 & 4.61 & 3.411 & 3.422 & 159.871 \\
60 & 3.36 & 4.028 & 4.035 & 188.789 \\
\hline
\end{tabular}

\section{Rim Interaction}

The nature of the impact between the ball and the far rim is crucial in arriving at a valid value of $V$. There are two aspects which seem to be relevant. The first of these (Aspect 1) concerns whether the ball and the rim grip each other upon contact or slip against each other. There is no other condition possible - either they grip or they slip. Even when slipping is in effect there is another consideration and that is the value of the coefficient of friction, $\mu$. When two bodies slide against each other the friction force depends directly upon the value of $\mu$. The frictional force plays a role in the modeling of the impact when there is slippage. The second aspect (Aspect 2) concerns the extent of bounce that takes place when the ball hits the far rim. This aspect is quantified by $e$, the coefficient of restitution. Holmes (1991) takes a stand in these matters. For Aspect 1 Holmes (1991) advocates grip rather than slip, and for Aspect 2 it declares that $e=0$ - that is, there is no bounce. This latter assertion is subject to scrutiny since instances of putts bouncing off the far rim have been observed both in practice and on televised golf tournaments. In contrast to Holmes' (1991) position that the ball neither slips nor bounces upon hitting the far rim, the current authors have observed bouncing and see no reason why slipping is not also possible. The degree to which these two aspects occur may not be constant since ambient conditions could influence the values of $\mu$ and $e$. The lubricating effect of moisture might lead to slippage, while under dry conditions the same conditions might result in gripping.

Since both aspects have opposing limits it is clear that there are four alternatives. These are denoted by Cases A, B, $\mathrm{C}$, and $\mathrm{D}$, and their meaning is given in Table 3 . These cases will be treated in the following two sections. 
Table 3. Definition of what is meant by Case A, Case B, Case C, and Case D.

\begin{tabular}{ccc}
\hline Aspect1 & Aspect2 & Case \\
\hline Slip & No bounce & $\mathrm{A}$ \\
Slip & Bounce & $\mathrm{B}$ \\
Grip & No bounce & $\mathrm{C}$ \\
Grip & Bounce & $\mathrm{D}$ \\
\hline
\end{tabular}

\section{Case A and Case B}

Cases A and B are treated together by using the results given by Cross (2015). Cross is an academic physicist who conducts research in sports science. Specifically, as many sports are played with balls, his interest gravitated toward the technical aspects of bouncing balls (Cross, 1999; 2002; 2010). In studying the mathematics of a bouncing ball, Cross develops equations which are relevant to Cases $\mathrm{A}$ and $\mathrm{B}$, but do not apply to Cases $\mathrm{C}$ and D. We retain most of Cross's notation except for substituting $\xi$ for $\mathrm{x}$, and $\eta$ for $\mathrm{y}$. This is done since $\mathrm{x}$ and $\mathrm{y}$ are already in use with different meaning. The ball is envisioned to strike the $\xi$-axis with a descending blow and then to rebound. The subscript 1 applies to conditions just prior to the ball striking the $\xi$ - axis, while the subscript 2 refers to conditions just after contact. The ball's trajectory makes an angle of $\theta_{l}$ with the $\xi$ - axis just before contact, and just after contact it rebounds so that its trajectory makes an angle $\theta_{2}$ with the $\xi$ - axis. In the present situation, the $\xi-$ axis is taken to be the kissing tangent line to the ball at the point where the ball and the far rim meet. This is pictured in Figure 2. The $\eta$ - axis is perpendicular to the $\xi$-axis, the positive portion of which starts at the contact point and extends through the center of the ball and beyond.

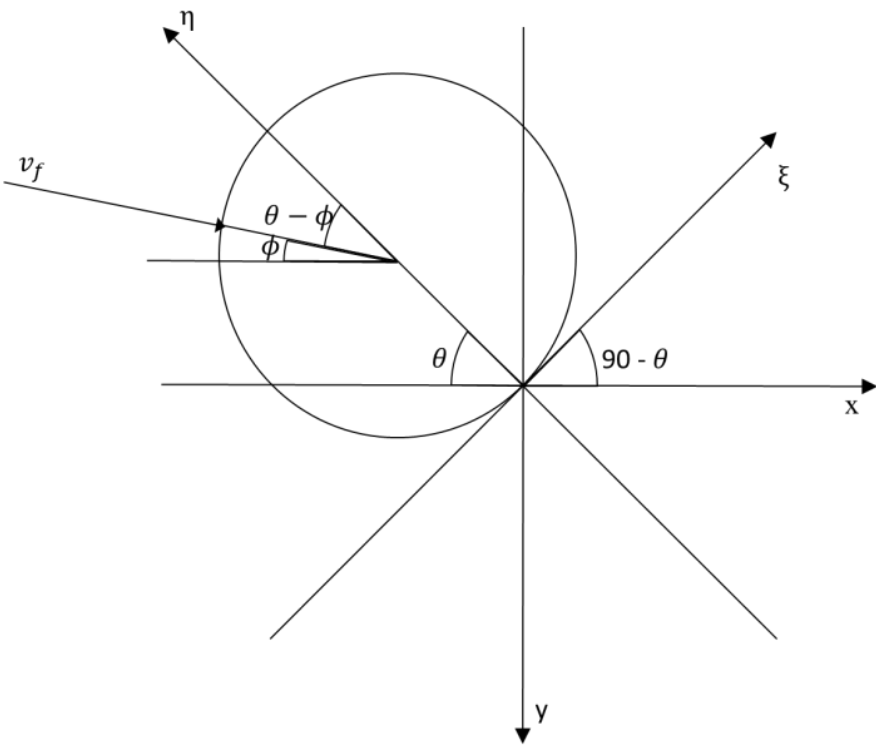

Figure 2. An enlargement showing the ball approaching from the left as it hits the far rim. Important angles are noted along with the $\mathrm{x}-\mathrm{y}$ coordinates and the $\xi-\eta$ coordinates.

After changing the independent variables in the manner just described, Cross's (2015) equations for a bouncing ball become

$$
v_{\xi 2}=v_{\xi 1}-\mu(1+e) v_{\eta 1}
$$

And

$$
v_{\eta 2}=e v_{\eta 1}
$$

Where $v_{\xi}$ and $v_{\eta}$ denote velocity components referred to the respective axes. In the present instance

$$
v_{\xi 1}=v_{f} \sin (\theta-\phi)
$$

and

$$
v_{\eta 1}=v_{f} \cos (\theta-\phi)
$$

Unquestionably, $v_{\eta 1}$ should be negative, but as a matter of convenience Cross (2015) specifies that it is to be taken as positive. Upon incorporating (14) and the trigonometric identities for $\sin (\theta-\phi)$ and $\cos (\theta-\phi)$ one obtains

$$
v_{\xi 1}=v(\sin \theta-\cos \theta \tan \phi)
$$


and

$$
v_{\eta 1}=v(\cos \theta+\sin \theta \tan \phi)
$$

Note that

$$
\tan \theta_{2}=v_{\eta 2} / v_{\xi 1}
$$

Combination of (16), (17), (20), (21), and (22) yields

$$
\tan \theta_{2}=e(\cos \theta+\sin \theta \tan \phi) /[\sin \theta-\cos \theta \tan \phi-\mu(1+e)(\cos \theta+\sin \theta \tan \phi)]
$$

The rebound angle $\theta_{2}$ is referred to the $\xi$ - axis. When the same rebound is referred to the horizontal axis, the rebound angle becomes $\theta_{2}+90-\theta$ degrees. If the rebound is to be straight up (and hence be on the verge of escaping) this latter angle must be 90 degrees. This requires that $\theta_{2}$ and $\theta$ be equal. Upon setting $\theta_{2}$ equal to $\theta$ in (23) followed by rearrangement one obtains

$$
\tan \theta(\tan \theta-\tan \phi)-(1+\tan \theta \tan \phi)[e+\mu(1+e) \tan \theta]=0
$$

Upon using (12) to provide values of $\tan \phi,(24)$ may be solved numerically for $\theta$ for specified values of $e$ and $\mu$. Some values of $\theta$ are tabulated in Table 4. For those values of $\theta$, (13) may be used to find the corresponding values of $V$. These numbers are also shown in Table 4.

Table 4. The first table gives value of $\theta$ (expressed in degrees) obtained by solving (24) using selected values of e and $\mu$. The second table presents the corresponding values of $\mathrm{V}$ (expressed in $\mathrm{m} / \mathrm{s}$ ) as obtained from (13). The columns headed by $\mathrm{e}=0$ correspond to Case $\mathrm{A}$. The remaining columns are for Case B.

\begin{tabular}{ccccc}
\hline & $e=0$ & $e=0.1$ & $e=0.2$ & $e=0.3$ \\
\hline$\mu=0$ & $\theta=18.41$ & $\theta=26.36$ & $\theta=31.19$ & $\theta=34.80$ \\
$\mu=0.1$ & 22.38 & 29.57 & 34.20 & 37.70 \\
$\mu=0.2$ & 26.29 & 32.81 & 37.22 & 40.61 \\
$\mu=0.3$ & 30.08 & 36.02 & 40.20 & 43.45 \\
\hline$\mu=0$ & $e=0$ & $e=0.1$ & $e=0.2$ & $e=0.3$ \\
\hline$\mu=0.1$ & $V=1.607$ & $V=1.806$ & $V=1.958$ & $V=2.092$ \\
$\mu=0.2$ & 1.699 & 1.904 & 2.068 & 2.215 \\
$\mu=0.3$ & 1.804 & 2.016 & 2.193 & 2.353 \\
\hline
\end{tabular}

\section{Case $C$ and Case $D$}

Case C stipulates that upon contact the ball grips the far rim and that it does not bounce. This is the situation envisioned by Holmes (1991). Since there is no bounce the ball relinquishes its linear motion. The same must be true of the rotary motion. The reason for this follows from the condition necessary for evaluating $V$. That condition requires that the ball be in a neutral state. That is, the ball must be on the border between escape and capture. If after initial contact the ball were to have even a slight rotation to the right (that is, clockwise) it would surely escape. If it had a rotation to the left it would be captured. Hence, to be in the neutral state rotation must stop.

The neutral state is only possible if the moment urging rotation in one direction is exactly countered by an equal moment in the opposite direction.

The sudden stopping of linear motion gives rise to one of the moments - call it $\tau_{1}$. The tangential velocity rapidly goes from $v_{\xi 1}$ to zero. The acceleration involved is

$$
a_{\xi}=\left(0-v_{\xi 1}\right) / \epsilon
$$

where $\epsilon$ is the effective time required to bring about the change. The force required is

$$
F_{\xi}=m a_{\xi}
$$

and the associated moment is

$$
\tau_{1}=r F_{\xi}
$$

Combining (20) with (25), (26), and (27) gives

$$
\tau_{1}=m r v(\cos \theta \tan \phi-\sin \theta) / \epsilon
$$

The rotation of $\omega$ abruptly stops and involves an angular acceleration of

$$
\alpha=(0-\omega) / \epsilon
$$

The associated moment is

$$
\tau_{2}=I \alpha
$$


where $I$ is the moment of inertia of the ball. A reasonable value of $I$ is that for a uniform sphere

$$
I=(2 / 5) m r^{2}
$$

Combining (15) with (29), (30), and (31) gives

$$
\tau_{2}=-(2 / 5) m r v / \epsilon
$$

Since $\tau_{1}$ and $\tau_{2}$ are equal and opposite their sum must be zero. That result yields

$$
(2 / 5)=\cos \theta(\tan \phi-\tan \theta)
$$

Upon using (12), the numerical solution to (33) is $\theta=3.5177$ degrees. Then substitution of this value of $\theta$ into (13) produces $V=1.356 \mathrm{~m} / \mathrm{s}$. Case D allows for bounce. An exact analysis of this case is daunting due to our inability to develop equations which predict the angle of rebound $\theta_{2}$. Scrutiny of Case $\mathrm{A}$ and Case $\mathrm{B}$ suggests that an increase in bounce results in an increase of $V$. If this rule carries over to Case $\mathrm{C}$ and Case $\mathrm{D}$ then the values of $V$ for Case $\mathrm{D}$ should exceed the value of $V$ found for Case $C$ which was $1.356 \mathrm{~m} / \mathrm{s}$.

\section{Conclusion}

The primary goal of this study was to establish the critical value of the launch velocity, $V$. It is critical in the sense that it represents a point in the continuum of launch velocities which separates putts which are captured by the hole from putts which escape capture by jumping over it. Only putts directed along the diameter of the hole are involved. As a prelude to this main thrust we found that the attitude of the ball just prior to hitting the far rim (given by $\theta$ ), the slope of the terminal trajectory (given by $\tan \phi$ ), and the launch velocity (given by $v$ ) are related through (12) and (13). Furthermore, the terminal motion of the ball as symbolized by $v_{f}$ and $\omega$ are represented by (14) and (15). Selected values of these variables are displayed in Table 2.

The value of $V$ plays a role in theoretical studies of putting, see Holmes (1991), Penner (2002), and Mahoney, Connaughton, \& Jang (2016). The value adopted by these researchers is $1.626 \mathrm{~m} / \mathrm{s}$ (or rounded to 1.63 ) which is the number proposed by Holmes (1991). Because of its perceived importance, further study into the value of $V$ seemed appropriate.

Although $1.626 \mathrm{~m} / \mathrm{s}$ appears to be a usable value of $V$, we believe that it cannot be the result of the no bounce and no slip assumption. That corresponds to Case $C$ where we found $V=1.356 \mathrm{~m} / \mathrm{s}$. We have observed balls bouncing off the far rim, and we imagine that slipping is also possible when there is sufficient moisture to act as a lubricant. Table 4 follows from the equations of Cross (2015) and shows that when bounce and slip are allowed that values of $V$ depend strongly upon $e$ and $\mu$. It seems unlikely that $e$ and $\mu$ would always have the same value, hence it seems reasonable that $V$ would not be constant, but would depend upon ambient conditions. Increasing the value of $V$ would benefit golfers who wish to improve their putting proficiency since by doing so the chance of the ball jumping over the hole would be lessened. There seems to be no practical manner to preferentially augment $V$ since golfers are enjoined to follow the rules and to play the course as they find it. Impractical means of increasing $V$ could involve increasing $D$ (the size of the hole), or decreasing $d$ (the diameter of the ball). Also, reference to Table 4 shows that any increase in $e$ (coefficient of restitution) or of $\mu$ (coefficient of sliding friction) would result in the sought-after increase in $V$. We offer no sure method for increasing these coefficients.

\section{References}

Alexander, D. L. \& Kern, W. (2005) Drive for show and putt for dough: An analysis of the earnings of PGA Tour golfers. Journal of Sports Economics, 6(1), 46-60.

Broadie, M. (2012). Assessing golfer performance on the PGA tour. Interfaces, 42(2), 146-165.

Cross, R. (1999). The bounce of a ball. American Journal of Physics, 67(3), 222-227.

Cross, R. (2002). Grip slip behavior of a bouncing ball. American Journal of Physics, 70(11), 1093-1102.

Cross, R. (2010). Enhancing the bounce of a ball. Physics Teacher, 48, 450-452.

Cross, R. (2015). Behaviour of a bouncing ball. Physics Education, 50(3), 335-341.

Holmes, B. W. (1991). Putting: How a golf ball and hole interact. American Journal of Physics, 59(2), 129-136.

Mahoney, J. F. (1982). Theoretical analysis of aggressive golf putts. Research Quarterly for Exercise and Sport, 53(2), 165-171.

Mahoney, J. F., Connaughton, D. P., \& Jang, W. (2016). Geometric aspects of strategic putting. International Journal of Golf Science, 5(1), 38-48.

Pelz, D. (2000). Dave Pelz's putting bible. New York, NY: Doubleday.

Penner, A. R. (2002). The physics of putting. Canadian Journal of Physics, 80(2), 83-96.

Wiren, G. (1992). Golf: Building a solid game. Englewood Cliffs, NJ: Prentice-Hall. 